\title{
Development of new instrumentation for epithermal neutron scattering at very low angles
}

\author{
M. Tardocchia, ${ }^{\mathrm{a}}$, A. Pietropaolo ${ }^{\mathrm{b}}$, C. Andreani ${ }^{\mathrm{b}}$, G. Gorini ${ }^{\mathrm{a}}$, E. Perelli-Cippo ${ }^{\mathrm{a}}$, \\ N.J. Rhodes ${ }^{\mathrm{c}}$, E.M. Schooneveld ${ }^{\mathrm{c}}$, R. Senesi ${ }^{\mathrm{b}}$ \\ aINFM, UdR Milano-Bicocca and Dipartimento di Fisica "G.Occhialini”, Università degli Studi di Milano-Bicocca, \\ Pizza Della Scienza 3, Milano 20126, Italy \\ ${ }^{\mathrm{b}}$ Dipartimento di Fisica, Università degli Studi di Roma Tor Vergata and INFM, UdR Roma Tor Vergata, Roma, Italy \\ ${ }^{\mathrm{c}}$ Isis Facility, Rutherford Appleton Laboratory, Chilton, Didcot, UK
}

Available online 12 August 2004

\begin{abstract}
New perspectives for epithermal neutron spectroscopy are opened up by the recent developments of Resonance Detectors (RD) for inverse geometry time-of-flight spectrometers at pulsed neutron sources. The RD is based on the combination of an analyser foil used as neutron-to-gamma converter and a suitable photon detector. Here, we report on the state of the RD which is based on a YAP scintillator viewing a natural uranium analyser foil. The response of the YAP detector to the radiative capture $\gamma$ emission from the uranium analyser foil has been characterized with a biparametric measurement of a reference $\mathrm{Pb}$ sample, which allowed simultaneous measurements of both neutron time-offlight and $\gamma$ pulse height (energy) spectra. The analysis of the $\gamma$ pulse height and neutron time-of-flight spectra permitted to identify the signal and background components. These measurements showed that a significant improvement in the signal-to-background ratio can be achieved by setting a lower level discrimination on the photon energy at about $600 \mathrm{keV}$. The first application of RD is the Very Low Angle Detector Bank (VLAD) which is planned to be installed in the next three years as an upgrade of the VESUVIO spectrometer, at the ISIS pulsed neutron source. VLAD will extend the $(q, \omega)$ kinematical to low wave vector transfers $\left(q<10 \AA^{-1}\right)$ coupled to high-energy transfers $(\hbar \omega>1 \mathrm{eV})$, which is still unexplored by neutron scattering experiments. The first measurements obtained on an ice sample with a VLAD prototype consisting of four RD units are presented here.
\end{abstract}

(C) 2004 Elsevier B.V. All rights reserved.

PACS: $29.30 . \mathrm{Hs} ; 29.30 . \mathrm{Kv} ; 29.40 . \mathrm{Wk} ; 61.12 . \mathrm{Ex}$

Keywords: Neutron spectroscopy; Epithermal neutron; Resonance detector

\footnotetext{
*Corresponding author. Tel.: + 39-02-6448-2326; fax: + 3902-6448-2367.

E-mail address: marco.tardocchi@mib.infn.it (M. Tardocchi).
}

\section{Introduction}

The recent developments of new instrumentation for inverse geometry time-of-flight spectrometers 
at pulsed neutron sources are motivated by the need to extend the kinematical region covered by neutron scattering experiments. In particular, new experimental studies in condensed matter systems, such as, for instance, high-energy excitations in magnetic systems and semiconductors [1] become possible by accessing the kinematical region of low wave vector transfer $\left(q<10 \AA^{-1}\right)$ coupled to high-energy transfer $(\hbar \omega>1 \mathrm{eV})$. Experiments in this kinematical region require the detection of epithermal neutrons in the $1-100 \mathrm{eV}$ energy range at scattering angles below $5^{\circ}[2]$.

Epithermal neutrons are routinely measured on the inverse geometry neutron spectrometer VESUVIO [3], installed at the ISIS pulsed neutron source. The neutron detection technique employed, namely the Resonance Filter (RF) technique, is based on ${ }^{6} \mathrm{Li}$ glass scintillators for detection of the scattered neutrons that are transmitted through an analyser foil [4]. The scattering timeof-flight spectrum at the final neutron energy specified by the capture resonance is obtained through the difference of the measurements with and without the analyser foil. The RF technique is effective for neutron energies in the $\mathrm{eV}$ range but it becomes inadequate above $10 \mathrm{eV}$ due to the $1 / \sqrt{ } E_{\mathrm{n}}$ dependence of the neutron capture cross-section of ${ }^{6} \mathrm{Li}$. For this reason, the Resonance Detector (RD) has been developed as an alternative detector. The $\mathrm{RD}$ consists of an analyser foil and a photon detector. Neutron detection is performed in a twostep process: (i) resonant neutron capture by an analyser foil $\left({ }^{\mathrm{A}} X\right)$ which strongly absorbs the scattered neutrons over a narrow energy interval; (ii) detection of the prompt radiative capture $\gamma$ rays following the radiative decay of the compound nucleus $\left({ }^{\mathrm{A}+1} X\right)$ to the ground state. The photon detector tags the arrival time of the absorbed neutron by detecting the photon cascade. The kinematics of the scattering event can thus be reconstructed through the time-of-flight technique.

In this work, we report on the state-of-the-art of the RD which, after few years of prototype development, has been brought to a satisfactory performance level. Results are presented on the characterization of the RD, as well as on the first RD measurements at very low scattering angles.

\section{The resonance detector}

The instrument developments and prototype tests have been performed on the VESUVIO neutron spectrometer [3] which operates at the ISIS spallation neutron source. In this instrument (Fig. 1), the incident neutron beam (n) impinges on the sample, placed inside an aluminium vacuum chamber, located at a distance $L_{0}=11.055 \mathrm{~m}$ from the moderator. The neutron beam is partly transmitted towards the beam dump, placed $5 \mathrm{~m}$ beyond the sample position, and partly scattered. Scattered neutrons of specific energies are detected by RD units placed at different locations around the sample. In the first example reported here, the sample was a $1 \mathrm{~mm}$ thick $\mathrm{Pb}$ metallic slab $\left(2.5 \times 3.5 \mathrm{~cm}^{2}\right)$ and the RD was located at a distance of $0.30 \mathrm{~m}$ from the sample, at a scattering angle of $90^{\circ}$. In a more recent experiment, four RDs were positioned in the forward direction to measure neutrons scattered from an ice sample.

The present choice of the analyser foil and photon detector combination for the $\mathrm{RD}$ is the result of a few years of development. The ideal analyser foil should have widely separated resonances in the $1-100 \mathrm{eV}$ energy range, with high intensity and narrow energy widths. ${ }^{238} \mathrm{U}$ is the best choice [5]; its radiative capture $\gamma$-ray emission consists of many peaks ranging from $11 \mathrm{keV}$ to $4.060 \mathrm{MeV}$ [6]. The ideal photon detector should have good efficiency to the prompt radiative capture $\gamma$-rays while being insensitive to other background radiations. The first promising results were obtained with Cadmium-Zinc-Telluride (CZT) semiconductor detectors, which allows room temperature operation with good photon

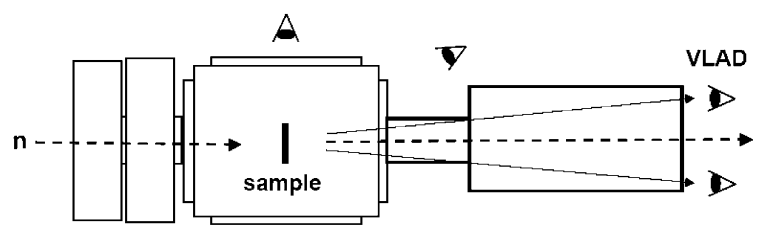

Fig. 1. Schematics of the experimental set-up of the measurement performed on the VESUVIO spectrometer. The figure shows the detector positions used for these measurements. 
detection efficiency in the range $20-200 \mathrm{keV}$. The results indicated that, even without any energy selection, the signal-to-background ratio exceeded the conventional ${ }^{6} \mathrm{Li}$-glass detectors $[7,8]$.

The RD performance was further improved with the use of $\mathrm{YAlO}_{3}$ (YAP) scintillators. YAP is an inorganic scintillator, non-hygroscopic, glasslike, with a high density $\left(5.55 \mathrm{~g} / \mathrm{cm}^{3}\right)$ but relatively low effective atomic number $(Z=36)$. The main characteristics of this scintillation material are a good light yield $(\approx 18,000$ photons/MeV) and a short decay time $(\tau=27 \mathrm{~ns})$ [9]. Its chemical composition is such that no neutron resonances are present in the energy range of interest $(1-200 \mathrm{eV})$. The detector was set-up to operate without any neutron or $\gamma$ shielding. The YAP crystal used was of cylindrical shape $(35 \mathrm{~mm}$ diameter by $6 \mathrm{~mm}$ thickness) and was coupled directly to a photomultiplier tube with bialkali photocathode. The thickness of the crystal was somewhat arbitrary but represents a compromise between the need for good efficiency to the $\gamma$ emission following neutron capture in the analyser foil and low neutron scattering in the crystal. Below $100 \mathrm{keV}$, the photoelectric interaction is the dominant process for energy deposition in the crystal and ensures $100 \%$ total absorption probability; Compton scattering provides a significant absorption probability (about 10\%) up to energies of several MeV [10].

The performance of a RD prototype composed of a YAP detector attached to a natural uranium analyser foil was assessed in biparametric measurements of a $\mathrm{Pb}$ polycrystalline sample [10]. The experiment allowed simultaneous measurements of both neutron time-of-flight (TOF) and $\gamma$ pulse height (energy) spectra. The TOF spectrum obtained projecting the biparametric data along the TOF axis for $\gamma$ energies above $600 \mathrm{keV}$ (Fig. 2) shows that $\mathrm{Pb}$ recoil peaks can be successfully distinguished form the background up to neutron energies of $190 \mathrm{eV}$. The comparison of the photon energy spectra corresponding to narrow TOF intervals around each neutron resonance and to wide off-resonance intervals has allowed to identify the main signal and background sources. The results indicate that the signal is predominantly made up of Compton events induced by radiative

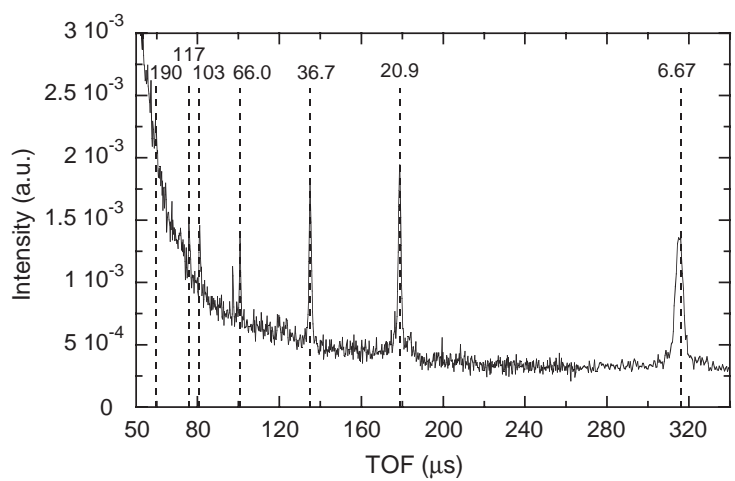

Fig. 2. Time-of-flight spectrum from the $\mathrm{Pb}$ sample obtained projecting the biparametric data along the time axis for $\gamma$ energies above $600 \mathrm{keV}$. The expected positions of the ${ }^{238} \mathrm{U}$ resonances are indicated by the dashed vertical lines and their energies (in eV) by the labels above the lines.

capture $\gamma$ rays which are not fully stopped in the detector. These signal events are superimposed on a background made of X-rays induced by nonresonant neutron absorption in ${ }^{238} \mathrm{U},{ }^{10} \mathrm{~B}$ radiative capture photons $\left(E_{\gamma}=478 \mathrm{keV}\right)$ and radioactivity of the analyser foil. The first two components are related in time to the neutron beam while the third one gives a flat contribution at all times. Boron is present in the wall of the experimental hall and in the beam dump.

The quality of the TOF measurements depends mainly on the intensity of the signal peaks relative to the background, i.e., the signal-tobackground ratio $S / B$. A significant improvement in the signal-to-background ratio has been achieved by selecting photon events with energies above $600 \mathrm{keV}$. This is shown in Fig. 3 for the resonance at $6.67 \mathrm{eV}$ where one observes an improvement in $S / B$ by about a factor 5 when the lower-level discrimination of the pulse height is raised from an equivalent $\gamma$ energy of about $60 \mathrm{keV}$ to $800 \mathrm{keV}$. The improvement is due to the discrimination of the background sources. Similar improvements have been observed for the other resonances. The results achieved with RD based on YAP detectors and uranium analyser foils have exceeded the conventional $\mathrm{RF}$ detectors even at neutron energies below $10 \mathrm{eV}$. 


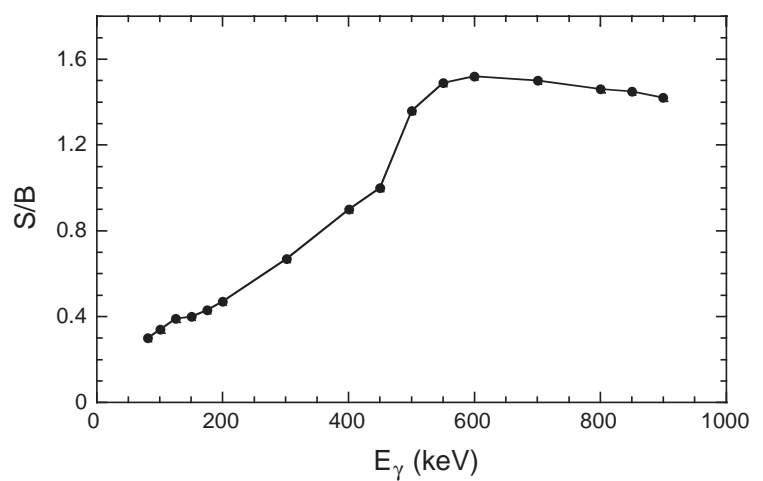

Fig. 3. Signal-to-background ratio $(S / B)$ versus the lower level discrimination on the $\gamma$ energy for the ${ }^{238} \mathrm{U}$ resonance located at $6.671 \mathrm{eV}$.

\section{Applications}

The first application of RD is the Very Low Angle Detector (VLAD) bank of the VESUVIO spectrometer which will cover the $1-5^{\circ}$ angular range at a distance of about $2 \mathrm{~m}$ from the scattering sample. The first step of the upgrade was the installation of a new vacuum tank, designed in order not to intercept the neutrons scattered at very low angles (see Fig. 1). The tank has a thin $(1 \mathrm{~mm})$ aluminium flange at the detector side. A detector bank prototype was built which consisted of four RD detectors. Each RD unit was made of two YAP crystals of trapezoidal shape (6 $\mathrm{mm}$ thick) enclosed in an aluminium case which acts as a light guide (Fig. 4). A $25 \mu \mathrm{m}{ }^{238} \mathrm{U}$ analyser foil was attached to each RD unit. Two detectors were positioned at fixed angular positions $\left(5^{\circ}\right)$ while the other two were placed on a movable frame adjustable between $2^{\circ}$ and $5^{\circ}$.

Measurements were taken from an ice sample at $270 \mathrm{~K}$. The four VLAD detectors covered the energy transfer range of $0.2-20 \mathrm{eV}$ and the wave vector transfer range of $3-10 \AA^{-1}$. The TOF spectrum, shown in Fig. 5 for the scattering angle of $2^{\circ}$, shows the hydrogen recoil peaks up to neutron energies of $117 \mathrm{eV}$. A preliminary analysis of the resonance at $6.67 \mathrm{eV}$ has indicated that one can derive quantitative values for the dynamical structure factor, $S(q, \omega)$, and the density of states, $g(\omega)$, of hydrogen in the sample using the same method for data analysis of previous experiments

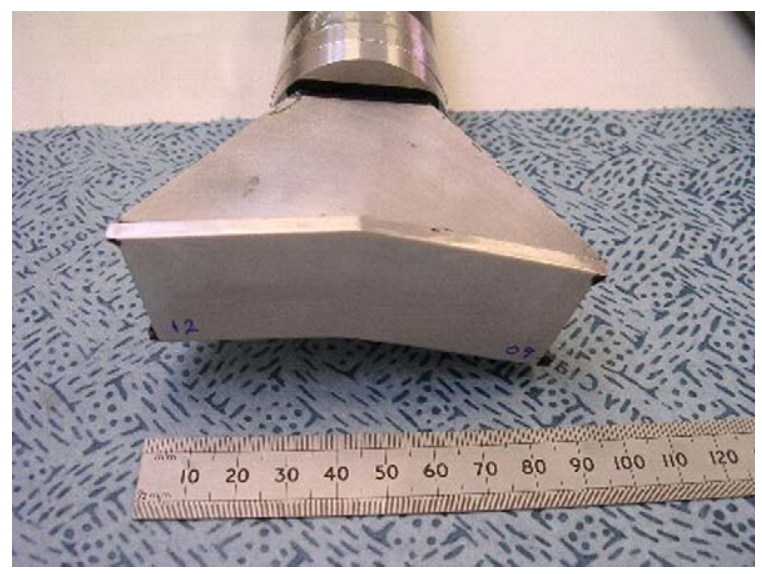

Fig. 4. Picture of the VLAD RD. The YAP detector is enclosed in an aluminium case attached to the photomultiplier tube.

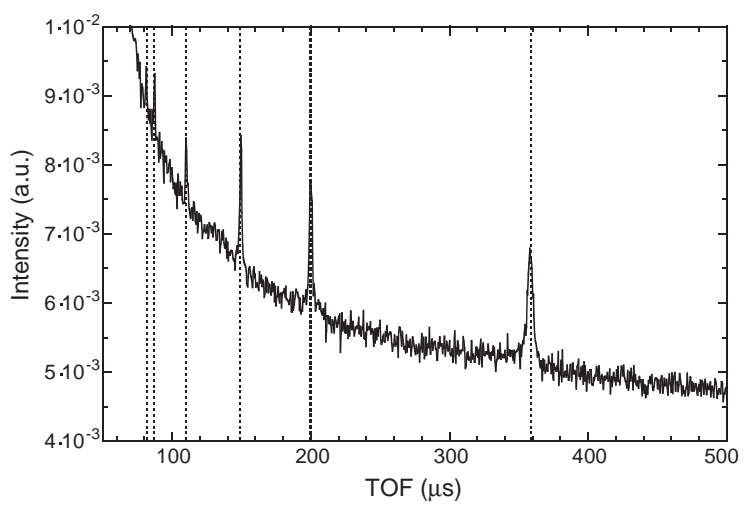

Fig. 5. Time-of-flight spectrum recorded from an ice sample in an $\mathrm{Al}$ cell in the time region $50-500 \mu \mathrm{s}$. The dashed lines indicate the $\mathrm{H}$ recoil peaks corresponding to the ${ }^{238} \mathrm{U}$ resonances.

performed on a different spectrometer [11]. These measurements are the first of its kind on an inverse geometry instrument.

\section{Conclusions}

New perspectives for epithermal neutron spectroscopy are opened up by the recent developments of RD at the VESUVIO inverse geometry neutron spectrometer. The RD performance achieved with the combination of a YAP scintillator and a natural uranium analyser foil has 
exceeded the conventional Li glass neutron detector. Characterization of the YAP response to the different signal and neutron sources has indicated that energy discrimination is an effective method to reduce the background interference and thus to improve the signal-to-background ratio in the TOF spectra. The first RD application is the Very Low Angle Detector Bank (VLAD) which will extend the kinematical region for neutron scattefing to low wave vector transfers $\left(q<10 \AA^{-1}\right)$ coupled to high-energy transfers $(\hbar \omega>1 \mathrm{eV})$. Accessing such kinematical regions will allow new experimental studies in condensed matter systems.

\section{Acknowledgements}

This work was performed with financial support by the European Community-Access to Research Infrastructure action of the Improving Human Potential Programme, within TECHNI project. We acknowledge Consiglio Nazionale delle Ricerche (CNR) - Italy for financial support for the experiments performed at the ISIS pulsed neutron source.

\section{References}

[1] R. Newport, et al., Nucl. Instr. and Meth. 224 (1984) 120.

[2] S. Imberti, et al., Resolution function of the VESUVIO spectrometer for high energy in elastic neutron scattering experiments, manuscript in preparation.

[3] R. Senesi, C. Andreani, Z. Bowden, D. Colognesi, E. Degiorgi, A.L. Fielding, J. Mayers, M. Nardone, J. Norris, M. Praitano, N.J. Rhodes, W.G. Stirling, J. Tomkinson, C. Uden, Physica B 276-278 (2000) 200.

[4] A.L. Fielding, J. Majers, Nucl. Instr. and Meth. A 48 (2002) 680.

[5] A. Pietropaolo, C. Andreani, A. D’Angelo, G. Gorini, S. Imberti, N. Rhodes, E. Schooneveld, R. Senesi, M. Tardocchi, Appl. Phys. A 74 (2002) S189.

[6] National Nuclear Data Center, Brookhaven National Laboratory, http://www.nndc.bnl.gov and Thermal Neutron Capture Home Page, Ernest O. Lawrence Berkeley National Laboratory, http://ie.lbl.gov/ng.html.

[7] C. Andreani, A. D'Angelo, G. Gorini, S. Imberti, A. Pietropaolo, N. Rhodes, E. Schooneveld, R. Senesi, M. Tardocchi, Appl. Phys. A 78 (2004) 903.

[8] M. Tardocchi, et al., Nucl. Instr. and.Meth. A. 526 (2004) 477.

[9] Bicron product catalogne, http://www.bicron.com.

[10] M. Tardocchi, et al., YAP scintillators for resonant detection of epithermal neutrons at pulsed neutron sources, Rev. Sci. Instr. 75(11) (2004), in press.

[11] C. Andreani, et al., A resonant detector for high-energy in elastic neutron scattering experiments, Appl. Phys. Lett. 29 Nov. (2004), in press. 